\title{
Nominal substitution in Meeteilon (Manipuri)
}

\author{
Huiningshumbam Surchandra Singh, P. Madhubala \\ Department of Linguistics, Manipur University, Canchipur, Imphal, India \\ Department of Linguistics, Manipur University, Canchipur, Imphal, India
}

\begin{abstract}
The present paper is an attempt to find out the characteristic features of Meeteilon linguistic element $m$ 'one' as substitute item in nominal substitution. The nominal substitute item ' $m$ ' is functioning as head of the nominal group bearing its own modifier. Another important feature of the substitute item is that it has been anaphoric. That means through the use of nominal substitution, a close relation is made between the presupposed noun and the nominal substitute ' $m$ '. This relation makes a cohesive discourse (both spoken and written). One important result of employing the nominal substitute item ' $m$ ' is to avoid the repetition of the same item. The nominal substitute item ' $m$ 'that is itself singular in number is used for both singular and plural presupposed noun. The nominal substitute item ' $m$ ' has the capacity to differentiate itself from the same item that is to be substituted in some sense. When the nominal substitution takes place, the substitute item ' $m$ ' replaces the head noun of the nominal group excluding the modifier. In such way, nominal substitution is now one of the cohesive devices of Meeteilon.
\end{abstract}

Key words: Anaphoric, Cohesive text, Head, Nominal substitution, Presupposed noun

\section{Introduction}

Substitution is one of the cohesive devices which are mainly used to make a cohesive text. It has three types namely, Nominal substitution, Verbal substitution and Clausal substitution. In fact, substitution takes place within the text. Simply, substitution means the replacement of an element by another one. In nominal substitution, a head noun of a nominal group is replaced by an element that functions as head of another nominal group. The present paper studies the nominal substitution in Meeteilon (Manipuri) and mainly focuses on the linguistic element $\mathbf{m}$ 'one' that functions as substitute item. It does not study any other linguistic items that function as substitute item in nominal substitution. It mainly focuses on the linguistic element $\mathbf{m}$ 'one' that functions as substitute item. Some important characteristic features of substitute item $\mathbf{m}$ 'one' in nominal substitution is given below:

i. it should be the head of the nominal group

ii. it should have its own modifier

iii. it must be anaphoric

iv. it should find its presupposed noun in the preceding text

v. it can never substitute for a proper name

\section{Nominal Substitution}

Substitution is the process that takes place within the discourse in which one item is replaced by another one. By nominal substitution, we mean substitution within the nominal group (Halliday and Hasan, 1976). It is a relation between the linguistic items, such as words or phrases. In terms of linguistic system, substitution is a relation on lexicogrammatical level, the level of grammar and vocabulary or linguistic forms (Halliday and Hasan, 1976). In one sense, it means to build up an anaphoric relation i.e., the substitute item refers back to the presupposed item in the preceding part of the discourse. A substitute is the sort of item that is employed to avoid the repetition of a particular item. In the case of nominal substitution, it means that the head of a nominal group is to be substituted by another item which would be the head of another nominal group. The substitute item has the same structural function as that for which it substitutes i.e., both the substitute item (that substitutes an element) and presupposed one (that is to be substituted) would be the head of their respective nominal groups. An illustrative example is given below:

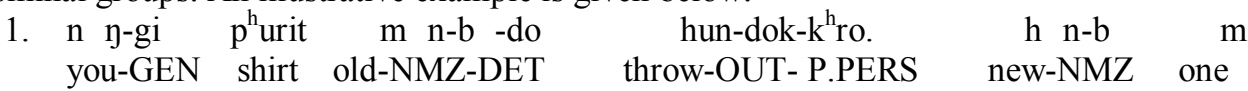

1 i-ro

buy-PERS

"Throw away your old shirt. Buy a new one."

In the above given example, $\mathbf{p}^{\text {h }}$ urit 'shirt' is the head of the nominal group $\mathbf{n}$ ygi $\mathbf{p}^{\mathrm{h}} \mathbf{u r i t} \mathbf{m} \mathbf{n b}$ 'your old shirt' and $\mathbf{m}$ 'one' is the head of the nominal group $\mathbf{h} \mathbf{n b} \mathbf{m}$ 'a new one'. Here, the lexical 
item $\mathbf{m}$ substitutes the noun $\mathbf{p}^{\mathrm{h}}$ urit which is in the preceding part of the discourse to avoid the repetition of the same item $\mathbf{p}^{\text {h }}$ urit in the second part of the utterance. The substitute item $\mathbf{m}$ always functions as head of a nominal group when it is used for substitution of a head noun. This substitute item can replace only for an item which is itself head of a nominal group i.e., $\mathbf{p}^{\mathrm{h}}$ urit. From the point of view of textual cohesion, of course, the substitute item $\mathbf{m}$ is anaphoric in the sense that it refers back to the referent $\mathbf{p}^{\mathbf{h}}$ urit that is in the preceding part of the discourse and hence, constituting a link between parts of the utterance especially between the presupposed item $\mathbf{p}^{\mathbf{h}}$ urit and the substitute item $\mathbf{m}$. In the light of the above given fact, the vast majority of instances of substitution are endophoric; and of these again, most of them are anaphoric (Halliday and Hasan, 1976). In fact, an occurrence of a substitute is a source of cohesion with what has gone before.

In nominal substitution process, there would have been presupposition. In a particular utterance the noun that is to be presupposed for a substitute item is found in the preceding utterance. But the presupposed noun of the substitute item $\mathbf{m}$ would always be a count noun. The substitute item $\mathbf{m}$ presupposes a noun that is to function as head in the nominal group.

2. h in u-sin si jamb $p$ t-le, $\mathrm{k}^{\mathrm{h}} \mathrm{r} \quad \mathrm{p}$ t-to-b $\mathrm{m}$ mango-PL this most rot-PERF some rot-NEG-NMZ one

$\mathrm{k}^{\mathrm{h}}$ n-bi-r k-u choose-HON-DEIC-COM

"Most of the mangoes are rotten. Please choose not so rotten one"

The substitute item $\mathbf{m}$ 'one' in the above example is presupposing the noun $\mathbf{h}$ in usin 'mangoes' which is itself plural in number in the preceding utterance and the presupposed noun is a count noun. It functions as head in the nominal group. The substitute item may differ from the presupposed item in number. In the above given example, the substitute item $\mathbf{m}$ is singular number whereas the presupposed item $\mathbf{h}$ in usin is plural in number. One important thing to bear in mind is that the substitute item $\mathbf{m}$ is itself singular number and it has no plural form. The substitute item $\mathbf{m}$ is used for both singular and plural presupposed item. In some context the modifier $\mathbf{k}^{\mathbf{h}} \mathbf{r}$ 'some' can be used as plural nominal substitute item. But it is not studied in this present paper.

The substitute item $\mathbf{m}$ carries over only the head noun of a nominal group. It does not carry over any modifying element by which this may have been accompanied. In other words, nominal substitution is done for the head noun only, not for any modifying element of that head. In nominal substitution, a substitute item is a carrier of some information that differentiates the instance in which it occurs from the instance to which it relates by cohesion.

3. ima-n $\quad k^{\text {hao }} k^{\text {h }} \mathrm{r} \quad \mathrm{g}$ ray $\quad 1$ i-r k-p -ni. pikp

mother-NOM bag some yesterday buy-DEIC-NMZ-COP small

$\mathrm{m} \quad 1$ u-ro

one take-PERS

"My mother bought some bags yesterday. Take a small one."

From the above given example it has been clearly seen that the substitute item $\mathbf{m}$ replaces the head noun $\mathbf{k}^{\mathbf{h}} \mathbf{a o}$ 'bag' of the nominal group $\mathbf{k}^{\mathbf{h}} \mathbf{a o} \mathbf{k}^{\mathbf{h}} \mathbf{r}$ 'some bags' excluding the modifier $\mathbf{k}^{\mathbf{h}} \mathbf{r}$ 'some'. The substitute item has its own modifier pikp 'small'. The substitute item $\mathbf{m}$ denotes the item to be quite different from the presupposed item $\mathbf{k}^{\mathbf{h}} \mathbf{a o}$ in the preceding text. The substitute item $\mathbf{m}$ denotes a bag which is the smallest one. In this way the substitute item $\mathbf{m}$ is the carrier of some information which has this differentiating function. Such differentiation happens mainly due to the presence of modifiers. Thus, every occurrence of a nominal substitution requires modifier to give such a differentiation.

In the case of the nominal substitution, the substitute item $\mathbf{m}$ 'one' carries the defining modifier (that defines an element) like pikp 'small' in the above example (3) and can, therefore, be thought of as a carrier of such defining elements. When the substitute item $\mathbf{m}$ carries over the head noun $\mathbf{k}^{\mathbf{h}} \mathbf{a o}$, its modifier $\mathbf{k}^{\mathbf{h}} \mathbf{r}$ is left over. Such item that is not carried over is called Repudiated element. In this sense, the process of defining has the effect of repudiating whatever is not carried over in the presupposition: the new definition is contrastive with respect to the original one. The substitute item $\mathbf{m}$ can never substitute for a proper name because a proper name is already fully defined as unique, and there is no way of adding to or altering the definition.

In the example given in (3), the modifier $\mathbf{k}^{\mathbf{h}} \mathbf{r}$ 'some' is repudiated when substitution takes place in the following part of the text. With the help of the new modifier pikp 'small', the substitute item can give a new definition which is contrastive with the original or preceding one $\mathbf{k}^{\mathbf{h}} \mathbf{a o}$ 'bag' i.e., the substitute item $\mathbf{m}$ with its own modifier pikp is differentiating from the presupposed noun in the sense that the substitute item means the 'smaller bag' which is quite different from the other bags mentioned in the preceding part of the text.

$$
\begin{aligned}
& \text { 4. } \mathrm{n} \quad \mathrm{y} \quad \mathrm{y} \text { ran-gi } \mathrm{t}^{\mathrm{h}} \text { udok-tu ninsin-li-b -ra? } \mathrm{m} d \mathrm{du} \\
& \text { you yesterday-OF incident-DET remember-CONT-NMZ-INT that } \\
& \text { kib } \quad m-d i \quad \text { oi }
\end{aligned}
$$


horrible one-EMPH be

"Do you remember yesterday's incident? That was a horrible one."

In the above example, it has been seen that by using the substitute item $\mathbf{m}$ with its own modifier (adjective) kib 'horrible' a specification is added to the presupposed item $\mathbf{t}^{\mathbf{h}}$ udok 'incident' and it also gives a redefining of the incident in the sense that what kind of incident was happening.

In nominal substitution, the presupposed item, if it is to be made explicit, replaces the presupposing one as in English example:

5. "Mummy will you buy me a bus? I want that red one."

Here, 'one' is the substitute item presupposing the noun 'bus' in the preceding text. The explicit form of the substitute item is made by replacing the substitute item 'one' by the presupposed item 'bus' as following :

6. "Mummy will you buy me a bus? I want that red bus."

And not as "I want that red one bus". As 'one' functions as numeral in the nominal group, it would precede the epithet 'red' as in the example (6).

In the case of Meeteilon (Manipuri), the presupposed item can replace the substitute item in its explicit form as in

7. "n ggi $\mathrm{p}^{\mathrm{h}}$ urit $\mathrm{m} \mathrm{nb}$ do hundokk ${ }^{\mathrm{h}}$ ro. $\mathrm{h}$ nb $\mathrm{m} 1$ iro."

The explicit form of the above given example can be given as following:

8. " $n$ ngi $\mathrm{p}^{\mathrm{h}}$ urit $\mathrm{m} \mathrm{nb}$ do hundokk ${ }^{\mathrm{h}}$ ro. $\mathrm{h}$ nb $\mathrm{p}^{\mathrm{h}}$ urit $\mathrm{m} 1$ iro."

In this sentence, it has been clearly seen that there has been the same item ' $\mathbf{m}$ ' as numeral because the head of the nominal group $\mathbf{h} \mathbf{n b} \mathbf{p}^{\mathbf{h}} \mathbf{u r i t} \mathbf{m}$ 'a new shirt' is $\mathbf{p}^{\mathbf{h}}$ urit 'shirt'. The presupposed noun ' $\mathbf{p}^{\mathbf{h}}$ urit' replaces the substitute item ' $\mathbf{m}$ ' when it is made explicit. In Meeteilon (Manipuri), the numerals follow the noun. One thing we can see in the above example (8) is that if the head noun $\mathbf{p}^{\mathbf{h}}$ urit 'shirt' of the nominal group $\mathbf{h} \mathbf{n b} \mathbf{p}^{\mathbf{h}} \mathbf{u r i t} \mathbf{m}$ 'a new shirt' is omitted, the remaining constituent in the nominal group is

h nb m 'a new one'.

Then the head of the nominal group will be $\mathbf{m}$ 'one' i.e, the head noun $\mathbf{p}^{\mathbf{h}} \mathbf{u r i t}$ 'shirt' is substituted by the element $\mathbf{m}$ 'one' because the same function done by the noun $\mathbf{p}^{\mathbf{h}} \mathbf{u r i t}$ can be done by $\mathbf{m}$ as substitute item. As the element $\mathbf{m}$ functions as head replacing the noun $\mathbf{p}^{\mathbf{h}}$ urit of the nominal group, the process involved here is the nominal substitution. The main distinction between the substitute item ' $\mathbf{m}$ ' and the numeral ' $\mathbf{m}$ ' is that the first one can function as head of a nominal group whereas the later one functions as modifier of the head noun of the nominal group.

\section{Conclusion}

The present paper has given a little hint on the use of the nominal substitution in Meeteilon (Manipuri) discourse. In this paper $\mathbf{m}$ 'one' has been studied as one of the nominal substitute items with supporting criteria of a nominal substitution. It has been really confirmed from the present study that nominal substitution is one of the cohesive devices employed in building up a cohesive discourse. One of the important results of using nominal substitution in a cohesive discourse is to avoid the repetition of the same item. Avoidance of such repetition can make a speaker or writer free from giving monotonous speech or writing to the listeners or readers. Besides this, it takes part in making a meaningful text (both spoken and written).It has become interesting to realise that such substitution contributes a lot to a cohesive discourse in Meeteilon. More exploration is required to find out many other linguistic elements that are used as substitute items in Meeteilon. Thus, it has become necessary to carry out such study for doing analysis of Meeteilon discourse.

\section{References}

[1] M.A.K. Halliday and Ruqaiya Hasan, Cohesion in English (London: Longman, 1976)

[2] P.Baker and S.Ellece, Key Terms in Discourse Analysis (London: Continuum, 2011)

[3] D.N.S. Bhat and M.S. Ningomba,Manipuri Grammar (Mysore: CIIL, 1995)

[4] Teun A.van Dijk (ed) Handbook of Discourse Analysis Vol.2. (US: Academic Press Inc., 1985)

[5] D. Blakemore, Understanding Utterances (Oxford: Blackwell, 1992)

[6] J. Cutting, Pragmatics and Discourse (London: Routledge, 2002)

[7] G. Brown and G.Yule, Discourse Analysis (Cambridge: Cambridge University Press, 1983)

[8] G. Yule, Pragmatics (New York: Oxford University Press, 1996)

[9] M.Coulthard, An Introduction to Discourse Analysis (London: Longman, 1986)

[10] J. Mey, Pragmatics: An Introduction (Oxford: Blackwell, 1994) 